\title{
A six-genotype genetic prognostic model for papillary thyroid cancer
}

\author{
Xiaopei Shen, Rengyun Liu and Mingzhao Xing \\ Laboratory for Cellular and Molecular Thyroid Research, Division of Endocrinology, \\ Diabetes \& Metabolism, Department of Medicine, Johns Hopkins University School of Medicine, \\ Baltimore, Maryland, USA
}

Correspondence should be addressed to $M$ Xing

Email

mxing1@jhmi.edu

\begin{abstract}
A unique prognostic role of the genetic duet of BRAF V600E and TERT promoter mutations in papillary thyroid cancer (PTC) has been recently established, but the role of RAS mutation in this genetic interplay remains to be established. Using The Cancer Genome Atlas (TCGA) data of patients with PTC from 19 medical centers, we investigated the interactions among the three mutations in clinical outcomes of PTC. We found that $B R A F$ and RAS mutations were mutually exclusive, but both were associated with TERT promoter mutations, with the genetic duet of BRAFIRAS and TERT mutations occurring in $34 / 388(8.76 \%)$ patients. BRAF/RAS or TERT mutation had no or minimal effect alone, whereas coexisting BRAF/RAS and TERT mutations had a robust synergistic effect on poor clinicopathologic outcomes of PTC, including disease recurrence and patient mortality. For example, PTC recurrence rate was $52 \%$ with coexisting BRAF V600E/RAS and TERT promoter mutations vs $6.9 \%$ with no mutation, corresponding to a HR of $8.17(95 \% \mathrm{Cl}$ 3.09-21.58), which remained significant at 14.71 (95\% Cl 2.79-77.61) after adjustment for clinicopathologic factors and institution. BRAFIRAS mutation or TERT mutation alone minimally affected Kaplan-Meier patient survival curves, whereas the genetic duet was associated with a sharp curve decline. Thus, by confirming and expanding previous findings in single-institution studies, this multicenter data analysis establishes a sixgenotype genetic prognostic model for poor outcomes of PTC with a risk order of genetic duet of BRAF V600E/RAS mutation and TERT mutation $>>>>B R A F \mathrm{~V} 600 \mathrm{E}=T E R T$ mutation alone $>R A S$ mutation alone $=$ wild-type genes.
\end{abstract}

\author{
Key Words \\ - thyroid cancer \\ - TERT promoter mutation \\ - BRAF V600E mutation \\ - RAS mutation \\ - prognostic molecular \\ markers
}

Endocrine-Related Cancer (2017) 24, 41-52

\section{Introduction}

Thyroid cancer is a common endocrine malignancy, consisting mostly of papillary thyroid cancer (PTC) and follicular thyroid cancer (FTC), with the former accounting for nearly $90 \%$ of all thyroid malignancies (Siegel et al. 2015, Howlader et al. 2016). PTC can be further classified into several variant types, including mainly conventional PTC (CPTC), follicular-variant PTC (FVPTC) and tall-cell PTC (TCPTC), among which CPTC was the most common. $B R A F \mathrm{~V} 600 \mathrm{E}$ and RAS mutations have been well established as the main genetic drivers of thyroid cancer, particularly
PTC (Garcia-Rostan et al. 2003, Xing 2013). Following the initial report of two mutually exclusive TERT promoter mutations (chr5:1,295,228 C>T and chr5:1,295,250 C>T; termed C228T and C250T, respectively) in melanoma in 2013 (Horn et al. 2013, Huang et al. 2013), we reported their occurrence in thyroid cancer also (Liu et al. 2013), which has been widely confirmed (Alzahrani et al. 2016, Liu \& Xing 2016). This represents an exciting recent development in understanding the genetic mechanisms of thyroid cancer. Studies from us (Liu et al. 2014, Shi et al. 2015) and others 
(Alzahrani et al. 2016, Liu \& Xing 2016) have consistently shown a strong association of TERT promoter mutations with aggressive clinicopathologic outcomes of thyroid cancer, suggesting a prognostic role of TERT promoter mutations in this cancer.

In our initial study on TERT promoter mutations in thyroid cancer, we made an interesting observation of the association between BRAF V600E and TERT promoter mutations (Liu et al. 2013). Our subsequent studies on extended cohorts of patients with different ethnic backgrounds again showed this phenomena (Liu et al. 2014, Shi et al. 2015), which has now been widely confirmed (Liu \& Xing 2016). BRAF V600E, the most common oncogene in PTC (Xing 2005), has been shown to be associated with a poor prognosis of PTC (Xing 2007, Xing et al. 2013, 2015). We found the unique genetic duet of coexisting BRAF V600E and TERT promoter mutations to be even more robustly associated with aggressive clinicopathologic outcomes of PTC, including tumor recurrence and patient mortality (Xing et al. 2014a,b, Liu et al. 2016). The genetic duet of RAS and TERT promoter mutations was shown to be marginally associated with aggressiveness of FTC in relatively small cohorts (Muzza et al. 2015, Sohn et al. 2016, Song et al. 2016). The role of this genetic duet in PTC has not been established.

These previous studies on the coexisting BRAF V600E and TERT promoter mutations were virtually all singleinstitution based. The genetic duet of BRAF V600E and TERT promoter mutations was studied in PTC virtually always with $R A S$ mutations mixed in the study cohort. It is important and ideal to use multicenter studies to validate and establish an exclusive role of the genetic duet of BRAF V600E and TERT promoter mutations and also to investigate the role of the genetic duet of RAS and TERT promoter mutations in PTC. To this end, we conducted the present study using the unique multicenter cohort of PTC patients in The Cancer Genome Atlas (TCGA) database (Cancer Genome Atlas Research Network 2014) to comprehensively investigate the interactions among BRAF V600E, RAS and TERT promoter mutations in affecting the clinical outcomes of PTC.

\section{Materials and methods}

\section{Mutation and clinical information of the PTC patients in the TCGA database}

Whole-exon mutation data were obtained from the TCGA Genome Data Analysis Center (GDAC) firehose website (http://firebrowse.org/). Information on BRAF V600E and RAS (including NRAS, KRAS and HRAS) mutations was extracted from the data. For $R A S$ mutations, only missense mutations were included. TERT promoter mutation information was extracted from the TCGA thyroid cancer mark paper (Cancer Genome Atlas Research Network 2014), which included the TERT promoter mutation information from the Sanger sequencing and whole genome sequencing. A total of 388 patients with available information on both exon mutation and TERT promoter mutation were included for analyses in the present study. Clinical data of these patients were extracted directly from the TCGA Data Portal (https://tcga-data.nci.nih.gov/tcga/). The information on new tumor event, disease status and patient mortality was updated to the latest follow-up data (07-30-2015, v4.0).

\section{Definition of disease recurrence and patient mortality}

PTC recurrence was defined here as recurrent or persistent disease as defined previously (Xing et al. 2014a), which was identified based on the clinical information of new thyroid cancer tumor-related events and the tumor status during the follow-up time for each patient. Patients with available information for both tumor status and new tumor event status during the follow-up time were included. Patients with any of the three types of new tumor events, including locoregional recurrent tumor, distant recurrent tumor and biochemical recurrent tumor, were identified as having disease recurrence. The recurrence time was defined as the time from the initial treatment of the original tumor to the discovery of the tumor recurrence/ persistence. If a patient has multiple new thyroid tumor events, the recurrence time for this patient was defined as the recurrence time for the first new tumor event. If the tumor status was 'with tumor' at any follow-up times even if it represented a persistent disease but not new tumor event, it was also treated as disease 'recurrence' in this study and the earliest follow-up time for the persistent tumor status was used as the recurrence time. Patients who had no new thyroid tumor event and were in 'tumor-free' status were treated as having no disease recurrence. As a result, a final 306 cases out of the initially selected 388 patients were used for PTC recurrence analysis. For patient mortality, the follow-up time was defined as the period from the treatment of the initial thyroid cancer to the time of death of the patient as defined previously (Xing 2013).

Published by Bioscientifica Ltd. 


\section{Statistical analyses}

Comparisons of categorical variables were performed with either Pearson's chi-squared test or, for cases with small number, Fisher's exact test. Wilcoxon-MannWhitney test was used for continuous variables. Survival curves were plotted with the Kaplan-Meier method with log-rank statistical analyses. Cox proportional hazards regression was used to assess the hazard ratio (HR) of the risk of recurrence and mortality. Statistical significance was defined as two-sided $P$ values $<0.05$.

\section{Results}

\section{BRAF V600E, RAS and TERT promoter mutations and} their relationship in PTC in the TCGA database

From the TCGA thyroid cancer database (Cancer Genome Atlas Research Network 2014), we identified 388 PTC patients from 19 sources/medical centers (Supplementary Table 1 , see section on supplementary data given at the end of this article) with available information both on exon mutation and TERT promoter mutation and analyzed the genetic status for BRAF V600E, RAS and TERT promoter mutations. BRAF V600E was found in 226/388 (58.2\%) cases, including 183/271 (67.5\%) CPTC, 13/83 (15.7\%) FVPTC and 28/30 (93.3\%) TCPTC. RAS mutations were found in 49/388 (12.6\%) cases, including 21/271 (7.7\%) CPTC, 28/83 (33.7\%) FVPTC and 0/30 (0\%) TCPTC. TERT promoter mutations were found in 26/271 (9.6\%) CPTC, $5 / 83(6.0 \%)$ FVPTC and 7/30 (23.3\%) TCPTC, with an overall prevalence of 39/388 (10.1\%) in all PTCs. The 39 cases of TERT promoter mutations included $30 \mathrm{C} 228 \mathrm{~T}$ (76.9\%), 8 C250T (20.5\%) and 1 C228A (2.6\%). The relationship among BRAF V600E, RAS and TERT promoter mutations is illustrated in Fig. 1. A significant association of TERT promoter mutations with the BRAF V600E/RAS mutation was observed $(P=0.016)$. Because $B A R F$ V600E and $R A S$ mutations were mutually exclusive $(P<2.2 \mathrm{e}-16)$, we analyzed the relationship between TERT and $B R A F$ V600E or RAS mutations in the subsets of $R A S$ mutation- or $B R A F$ V600E mutation-negative patients, respectively. In the RAS mutation-negative patients, BRAF V600E mutation was still significantly associated with TERT promoter mutation $(P=0.019)$. In the relatively small number of $B R A F$ V600E mutation-negative patients, RAS mutations were marginally associated with TERT promoter mutation $(P=0.085)$. Overall, the majority $(34 / 39 ; 87.2 \%)$ of the patients with TERT promoter mutations had either $B R A F$ V600E or RAS coexisting mutations, and 34/388 (8.76\%) of all PTC harbored BRAF V600E/RAS and coexisting TERT promoter mutations.

\section{Impacts of BRAF V600E or TERT promoter mutation alone or in their coexistence on clinicopathologic outcomes of PTC in the TCGA data}

When dividing the TCGA PTC cohort into two groups (TERT promoter mutation-positive vs -negative groups) a strong association of TERT promoter mutation with multiple poor clinicopathologic outcomes was seen (Supplementary Table 2). Similar effects of TERT promoter mutations on clinicopathologic outcomes were seen when the analysis was performed only on CPTC (Supplementary Table 3). On Kaplan-Meier analyses by dividing the cohort into TERT promoter mutation-negative and -positive groups, TERT promoter mutation was associated with a significant decline in the patient survival curve (Supplementary Fig. 1A) and

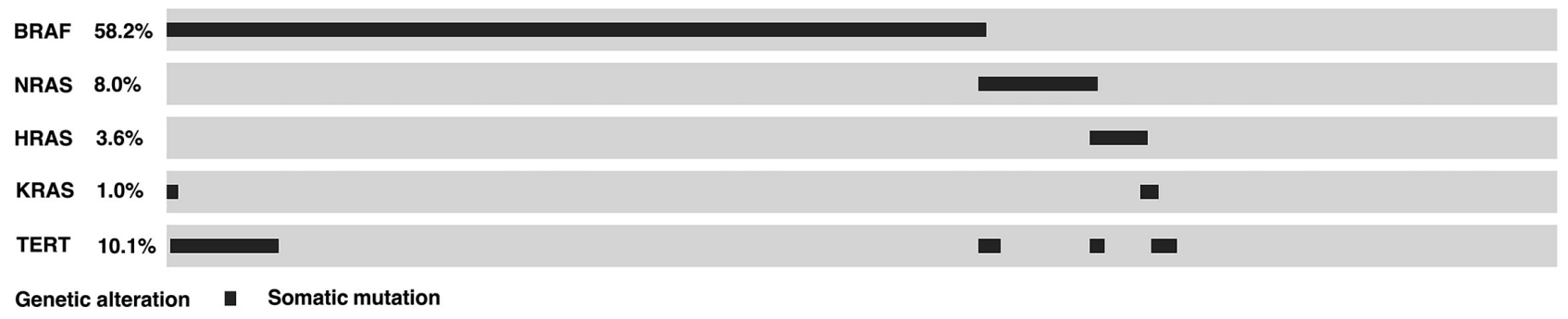

Figure 1

Gene-sample matrix of mutations on BRAF V600E, RAS and TERT promoter mutations. The matrix illustrates the genetic status of the 388 cases of papillary thyroid cancer (PTC) included in the present study from the TCGA database. Cases positive for the indicated gene mutations are marked with dark color. Several relationships among the different mutations are evident, including the mutual exclusiveness between BRAF V600E and RAS mutations, the association between BRAF V600E and TERT promoter mutations and the association between RAS and TERT promoter mutations in PTC. Occurrence of TERT promoter mutation alone is uncommon. Thirty-four of the $39(87.2 \%)$ patients positive for TERT promoter mutations have coexisting either BRAF V600E or RAS mutations and 34/388 (8.76\%) of all PTCs harbor the genetic duet of coexisting BRAF V600E/RAS and TERT promoter mutations. 
Table 1 Impacts of BRAF V600E or TERT promoter mutation alone or in their coexistence on clinicopathologic outcomes of PTC.

\begin{tabular}{l}
\hline Characteristic \\
\hline Sample size \\
Age at diagnosis, median (IQR) \\
(years) \\
Sex, male \\
Multifocality \\
Tumor size, median (IQR) (cm) \\
Extrathyroidal invasion \\
AJCC tumor stage \\
Stage I \\
Stage II \\
Stage III \\
Stage IV \\
Stage III + stage IV \\
AJCC_T \\
T1 \\
T2 \\
T3 \\
T4 \\
T3+T4 \\
AJCC_N, N1 \\
AJCC_M, M1 \\
Distant metastasis \\
Tumor recurrence \\
Mortality rate \\
Total follow-up, median (IQR) \\
(month) \\
\end{tabular}

\begin{tabular}{c}
\hline No mutation \\
\hline No. (percent) \\
\hline 109 \\
$46(33-59)$ \\
\\
$27 / 109(24.8)$ \\
$53 / 103(51.5)$ \\
$2.9(1.9-4)$ \\
$17 / 104(16.3)$ \\
\\
$66 / 108(61.1)$ \\
$18 / 108(16.7)$ \\
$19 / 108(17.6)$ \\
$5 / 108(4.6)$ \\
$24 / 108(22.2)$ \\
\\
$31 / 107(29.0)$ \\
$46 / 107(43.0)$ \\
$30 / 107(28.0)$ \\
$0 / 107(0)$ \\
$30 / 107(28.0)$ \\
$38 / 92(41.3)$ \\
$0 / 54(0)$ \\
$1 / 50(2)$ \\
$6 / 87(6.9)$ \\
$3 / 108(2.8)$ \\
$30(17-46)$ \\
\end{tabular}

\begin{tabular}{c}
\hline BRAF V600E o \\
\hline No. (percent) \\
\hline 197 \\
$46(34-54)$ \\
\\
$53 / 197(26.9)$ \\
$89 / 194(45.9)$ \\
$2.5(1.5-3.6)$ \\
$69 / 193(35.8)$ \\
\\
$115 / 196(58.7)$ \\
$15 / 196(7.7)$ \\
$47 / 196(24)$ \\
$19 / 196(9.7)$ \\
$66 / 196(33.7)$ \\
\\
$61 / 197(31)$ \\
$56 / 197(28.4)$ \\
$74 / 197(37.6)$ \\
$6 / 197(3)$ \\
$80 / 197(40.6)$ \\
$99 / 179(52.4)$ \\
$3 / 120(2.5)$ \\
$8 / 103(7.8)$ \\
$22 / 154(14.3)$ \\
$4 / 189(2.1)$ \\
$37(21-65)$ \\
\end{tabular}

\begin{tabular}{c}
\hline TERT mutation \\
\hline No. (percent) \\
\hline 5 \\
$49(37-68)$ \\
\\
$0 / 5(0)$ \\
$0 / 5(0)$ \\
$4(3.0-5.0)$ \\
$1 / 5(20)$ \\
\\
$3 / 5(60)$ \\
$1 / 5(20)$ \\
$0 / 5(0)$ \\
$1 / 5(20)$ \\
$1 / 5(20)$ \\
$1 / 5(20)$ \\
$2 / 5(40)$ \\
$1 / 5(20)$ \\
$1 / 5(20)$ \\
$2 / 5(40)$ \\
$3 / 5(60)$ \\
$0 / 4(0)$ \\
$0 / 4(0)$ \\
$1 / 5(20)$ \\
$1 / 5(20)$ \\
$13(6-28)$ \\
\end{tabular}

\begin{tabular}{l} 
only \\
\hline 0.52 \\
0.34 \\
0.06 \\
0.68 \\
1 \\
0.31 \\
\\
1 \\
0.06 \\
\\
0.62 \\
0.64 \\
1 \\
0.33 \\
0.17 \\
0.15 \\
\end{tabular}

\begin{tabular}{ccc}
\hline \multicolumn{2}{c}{ BRAF+ TERT mutation } \\
\cline { 1 - 1 } \cline { 1 - 1 } No. (percent) & & $P$ \\
\cline { 1 - 1 } 28 & & \\
$66(59-73)$ & & $<0.001$ \\
& & \\
$9 / 28(32.1)$ & & 0.47 \\
$12 / 28(42.9)$ & & 0.52 \\
$3(2.4-4.2)$ & & 0.21 \\
$17 / 28(60.7)$ & & $<0.001$ \\
& & \\
$5 / 28(17.9)$ & & $<0.001$ \\
$2 / 28(7.1)$ & & \\
$10 / 28(35.7)$ & & \\
$11 / 28(39.3)$ & & \\
$21 / 28(75)$ & & $<0.001$ \\
& & \\
$4 / 28(14.3)$ & & $<0.001$ \\
$6 / 28(21.4)$ & & \\
$11 / 28(39.3)$ & & \\
$7 / 28(25)$ & & \\
$18 / 28(64.3)$ & & $<0.001$ \\
$17 / 27(63.0)$ & 0.052 \\
$2 / 19(10.5)$ & 0.07 \\
$3 / 17(17.6)$ & 0.047 \\
$7 / 19(36.8)$ & & 0.002 \\
$5 / 28(17.9)$ & & 0.010 \\
$43(16-55)$ & & 0.13 \\
& & \\
\hline & &
\end{tabular}

This table summarizes the analyses in 339 cases of patients with PTC negative for RAS mutation. AJCC_M refers to distant metastasis status at the diagnosis of thyroid cancer; M1, presence of metastasis. The group 'Distant metastasis' below the group 'AJCC_M' in the table refers to any distant metastasis, including distant metastasis at the diagnosis and distant metastasis discovered during the follow-up time, i.e., metastatic recurrence. AJCC, American Joint Committee on Cancer; IQR, interquartile range.

recurrence-free survival curve (Supplementary Fig. 1B). Similar results were obtained on the effect of TERT promoter mutations when only CPTC was analyzed (Supplementary Fig. 1C and D).

To examine the effects of individual and coexisting genetic events of BRAF V600E and TERT promoter mutations, we divided the patients into four genotypes (Table 1). In this analysis, to exclude the influence of $R A S$ mutations, we focused the analyses on the 339 RAS mutation-negative PTC patients. In comparison with the group negative for either mutation, BRAF V600E alone was significantly associated with extrathyroidal invasion, late disease stages III and IV, high tumor stages T3 and T4 and lymph node metastasis $(P<0.001, P=0.050,0.034$ and 0.040 , respectively), and TERT promoter mutation alone did not show a significant association with any clinicopathologic outcome. In contrast, coexistence of BRAF V600E and TERT promoter mutation was robustly associated with virtually all the high-risk multiple clinicopathologic characteristics with more profound significance, including distant metastasis, disease recurrence and patient mortality $(P=0.047,0.002$ and 0.010 , respectively). Similar robust synergistic effects of coexisting BRAF V600E and TERT promoter mutations were observed when only CPTC in the TCGA data was analyzed (Supplementary Table 4).

A recent study demonstrated a differential aggressiveness risk for the three major PTC variants in the order of TCPTC $>$ CPTC $\gg$ FVPTC (Shi et al. 2016). We observed here a similar distribution pattern of coexisting BRAF V600E and TERT promoter mutations in the three PTC variants, with a prevalence being 7/30 (23.3\%), $20 / 270(7.4 \%)$ and $1 / 83(1.2 \%)$ in TCPTC, CPTC and FVPTC (Supplementary Table 5), respectively, consistent with an aggressive role of the genetic duet.

Table 2 summarizes the hazard ratios (HRs) of the impacts of BRAF V600E and TERT promoter mutations on PTC recurrence in RAS mutation-negative patients. The HR for recurrence was not significant for BRAF or TERT promoter mutation alone but robustly significant for coexisting BRAF V600E and TERT promoter mutations. Specifically, patients harboring the genetic duet had the

Published by Bioscientifica Ltd. 


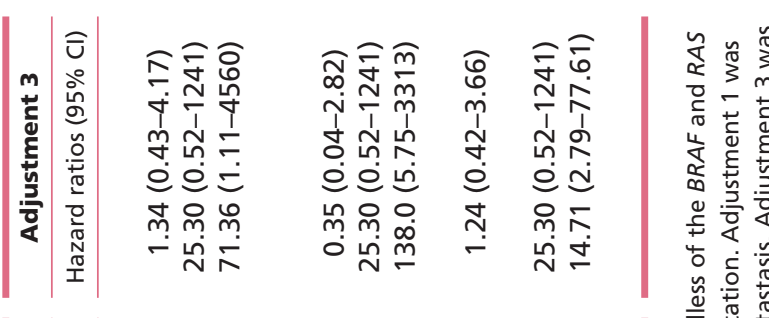

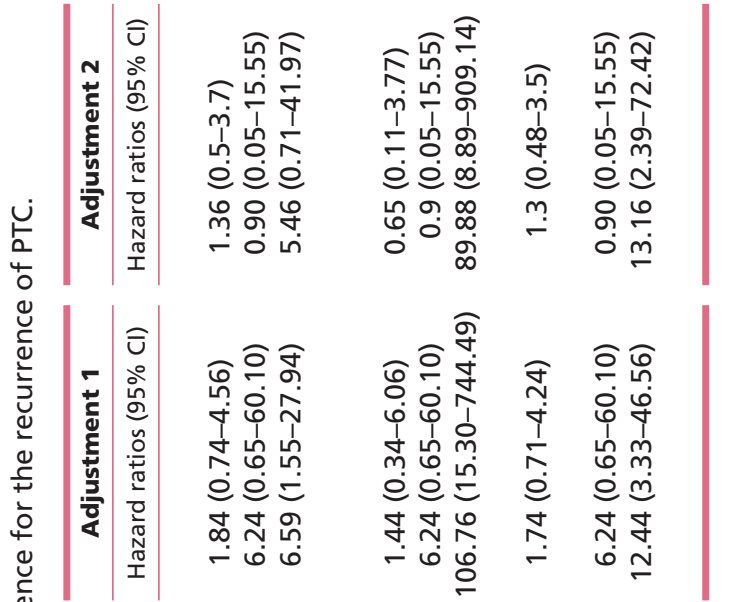

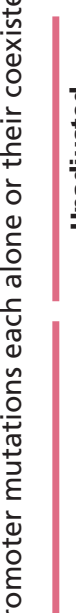

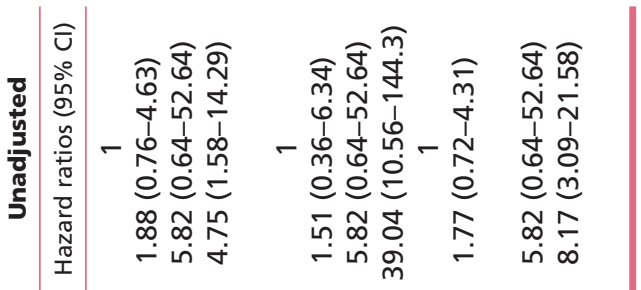

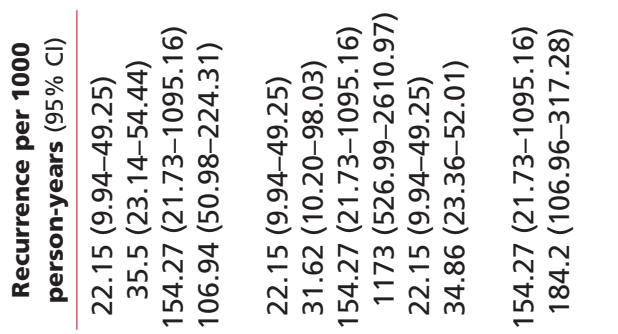

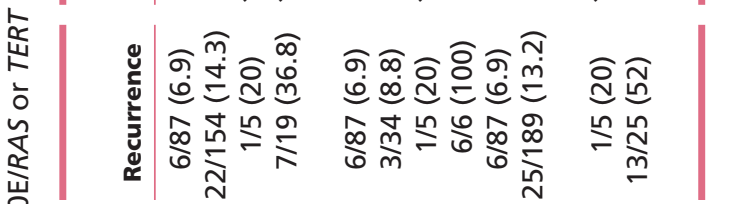

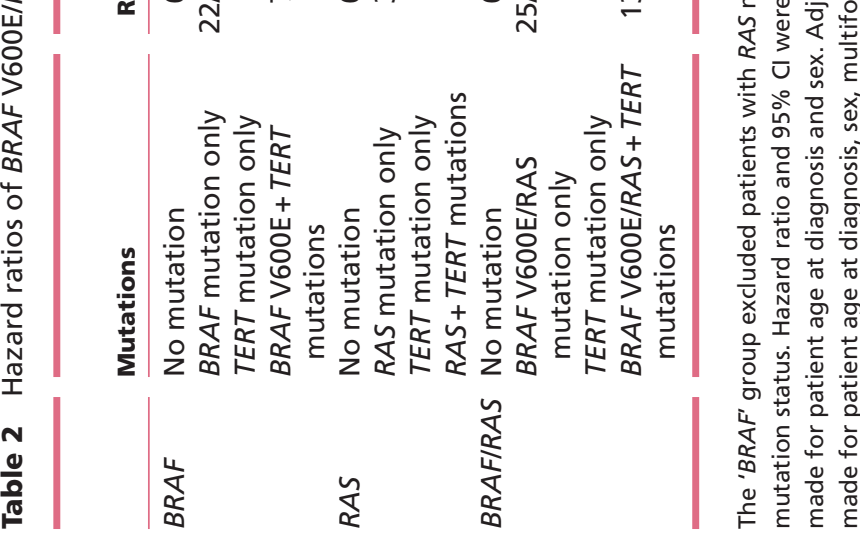


highest recurrence rate at $7 / 19$ (36.8\%), corresponding to 106.94 recurrences per 1000 person-years (95\% CI, 50.98-224.31), vs only 6/87 (6.9\%), corresponding to 22.15 recurrences per 1000 person-years (95\% CI, 9.94-49.25), in patients harboring neither mutation, with a HR of 4.75 (95\% CI, 1.58-14.29; $P=0.006)$.
A

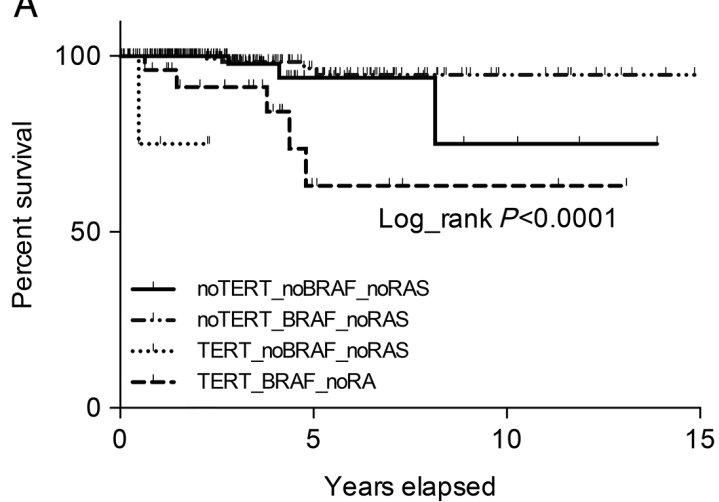

C

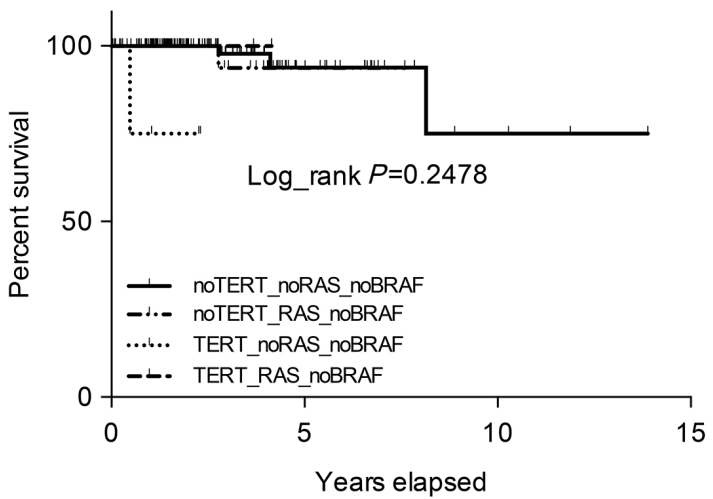

$E$

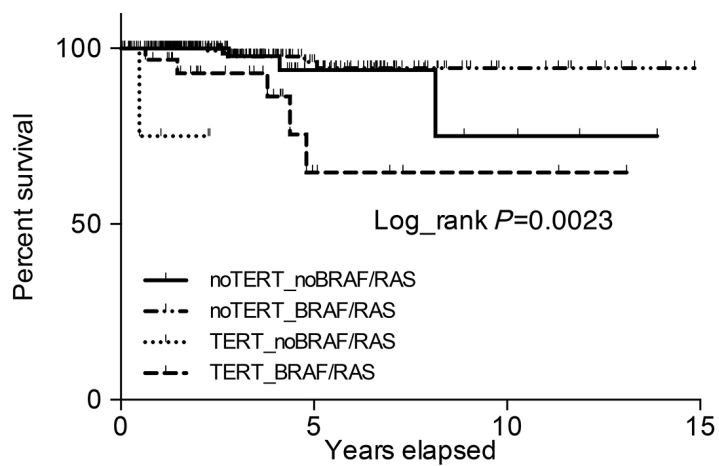

B

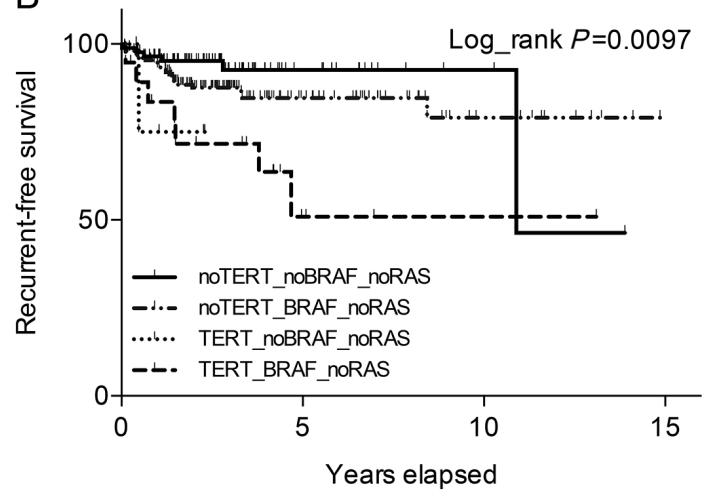

D

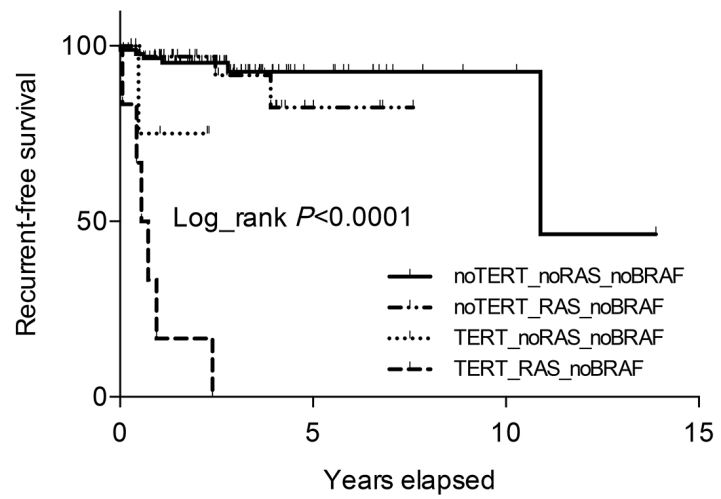

$\mathrm{F}$

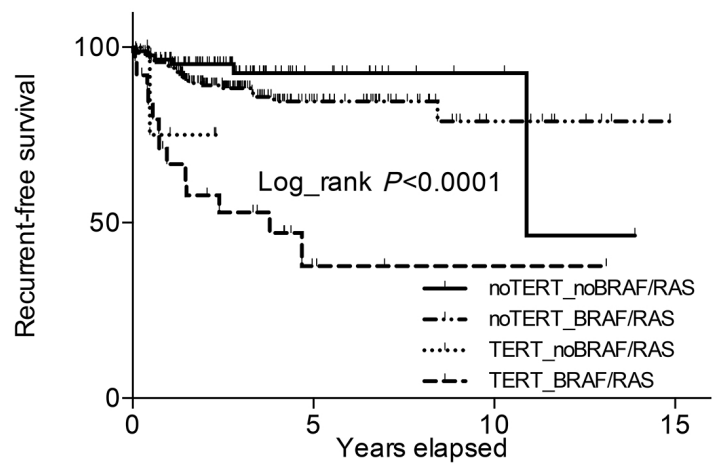

\section{Figure 2}

Kaplan-Meier analyses of the impacts of BRAF V600E, RAS or TERT promoter mutations or their coexistence on patient survival and disease recurrencefree survival of patients with papillary thyroid cancer (PTC) in the TCGA database. (A) Impacts of BRAF V600E or TERT promoter mutations or their coexistence on patient survival. (B) Impacts of BRAF V600E or TERT promoter mutations or their coexistence on PTC recurrence-free survival. The analyses in $\mathrm{A}$ and $\mathrm{B}$ were performed with exclusion of the cases positive for RAS mutations. (C) Impacts of RAS or TERT promoter mutations or their coexistence on patient survival. (D) Impacts of RAS or TERT promoter mutations or their coexistence on PTC recurrence-free survival. The analyses in C and D were performed with exclusion of the cases positive for BRAF V600E mutation. (E) Impacts of BRAF/RAS or TERT promoter mutations or their coexistence on patient survival. (F) Impacts of BRAFIRAS or TERT promoter mutations or their coexistence on PTC recurrence-free survival. The analyses in $E$ and $F$ were performed on the whole cohort of PTC patients without genetic-based exclusion. The log-rank $P$ value in each panel represents the comparison among the four groups globally. 
This HR remained significant at 6.59 (95\% CI, 1.55-27.94; $P=0.011)$ after adjustment for patient age and sex and marginally $(P=0.102)$ missed the significance after additional adjustment for tumor behaviors, but remained significant $(P=0.044)$ after further additional adjustment for institution (Table 2). Similar HR results for tumor recurrence were observed in the analysis of only CPTC (Supplementary Table 6).

We next performed Kaplan-Meier analyses of patient survival and recurrence-free survival by genotype in $R A S$ mutation-negative patients. Comparison of the four genotype groups globally revealed a significant difference in survival and recurrence-free survival $(P<0.0001$ and $P=0.0097$, Fig. $2 \mathrm{~A}$ and $\mathrm{B}$ ). As shown in Fig. $2 \mathrm{~A}$, in paired group comparison, compared with the group negative for either mutation, BRAF V600E mutation was not significantly associated with survival decline $(P=0.19)$, but coexisting BRAF V600E and TERT promoter mutations were significantly associated with a decline in the survival curve $(P=0.045)$. TERT promoter mutation alone was associated with a survival decline, but the number of cases was limited. For disease recurrence-free survival (Fig. 2B),
BRAF V600E or TERT promoter mutation alone had a modest effect ( $P=0.21$ and 0.08 , respectively), but their coexistence was robustly associated with a sharp decline in the recurrence-free survival curve $(P=0.002)$. These results were consistent with the synergistic effects of $B R A F \mathrm{~V} 600 \mathrm{E}$ and TERT promoter mutations on other clinicopathologic outcomes of PTC (Table 1). Similar results were obtained when Kaplan-Meier analyses were performed only on CPTC (Supplementary Fig. 2A and B).

\section{Impacts of RAS or TERT promoter mutation alone or their coexistence on clinicopathologic outcomes of PTC in the TCGA data}

We analyzed the impacts of RAS or TERT promoter mutation alone or in coexistence on clinicopathologic outcomes in the TCGA data (Table 3). To exclude the influence of $B R A F \mathrm{~V} 600 \mathrm{E}$, this analysis was focused on the 162 BRAF V600E mutation-negative PTC patients. Interestingly, in comparison with the group negative for either mutation, RAS mutation alone showed no adverse effect on any of the clinicopathological characteristics;

Table 3 Impact of RAS or TERT promoter mutation or their coexistence on clinicopathologic outcomes of PTC.

\begin{tabular}{l} 
Characteristic \\
\hline Sample size \\
Age at diagnosis, median (IQR) (years) \\
Sex, male \\
Multifocality \\
Tumor size, median (IQR) (cm) \\
Extrathyroidal invasion \\
AJCC tumor stage \\
Stage I \\
Stage II \\
Stage III \\
Stage IV \\
Stage III+stage IV \\
AJCC_T \\
T1 \\
T2 \\
T3 \\
T4 \\
T3+T4 \\
AJCC_N, N1 \\
AJCC_M, M1 \\
Distant metastasis \\
Tumor recurrence \\
Mortality rate \\
Total follow-up, median (IQR) (month)
\end{tabular}

\begin{tabular}{c}
\hline No mutation \\
\hline No. (percent) \\
\hline 109 \\
$46(33-59)$ \\
$27 / 109(24.8)$ \\
$53 / 103(51.5)$ \\
$2.9(1.9-4)$ \\
$17 / 104(16.3)$ \\
\\
$66 / 108(61.1)$ \\
$18 / 108(16.7)$ \\
$19 / 108(17.6)$ \\
$5 / 108(4.6)$ \\
$24 / 108(22.2)$ \\
\\
$31 / 107(29.0)$ \\
$46 / 107(43.0)$ \\
$30 / 107(28.0)$ \\
$0 / 107(0)$ \\
$30 / 107(28.0)$ \\
$38 / 92(41.3)$ \\
$0 / 54(0)$ \\
$1 / 50(2)$ \\
$6 / 87(6.9)$ \\
$3 / 108(2.8)$ \\
$30(17-46)$ \\
\end{tabular}

\begin{tabular}{|c|c|}
\hline \multicolumn{2}{|c|}{ RAS mutation only } \\
\hline No. (percent) & $P$ \\
\hline 42 & \\
\hline $41(32-50)$ & 0.1 \\
\hline 9/42 (21.4) & 0.83 \\
\hline $18 / 42(42.9)$ & 0.37 \\
\hline $2.3(1.8-2.9)$ & 0.13 \\
\hline $5 / 38(13.2)$ & 0.8 \\
\hline $31 / 42$ & 0.59 \\
\hline $4 / 42$ (9.5) & \\
\hline $6 / 42(14.3)$ & \\
\hline $1 / 42(2.4)$ & \\
\hline 7/42 (16.7) & 0.51 \\
\hline $16 / 42(38$ & 0.43 \\
\hline $18 / 42(42.9)$ & \\
\hline $8 / 42(19.0)$ & \\
\hline $0 / 42(0)$ & \\
\hline $8 / 42(19.0)$ & 0.61 \\
\hline $8 / 38(21.1)$ & 0.043 \\
\hline $0 / 26(0)$ & 1 \\
\hline $0 / 23(0)$ & 1 \\
\hline 3/34 (8.8) & 1 \\
\hline $1 / 42(2.4)$ & 1 \\
\hline $23(15-48)$ & 0.53 \\
\hline
\end{tabular}

\begin{tabular}{c}
\hline TERT mutation \\
\hline No. (percent) \\
\hline 5 \\
$49(37-68)$ \\
$0 / 5(0)$ \\
$0 / 5(0)$ \\
$4(3.0-5.0)$ \\
$1 / 5(20)$ \\
$3 / 5(60)$ \\
$1 / 5(20)$ \\
$0 / 5(0)$ \\
$1 / 5(20)$ \\
$1 / 5(20)$ \\
$1 / 5(20)$ \\
$2 / 5(40)$ \\
$1 / 5(20)$ \\
$1 / 5(20)$ \\
$2 / 5(40)$ \\
$3 / 5(60)$ \\
$0 / 4(0)$ \\
$0 / 4(0)$ \\
$1 / 5(20)$ \\
$1 / 5(20)$ \\
$13(6-28)$ \\
\end{tabular}

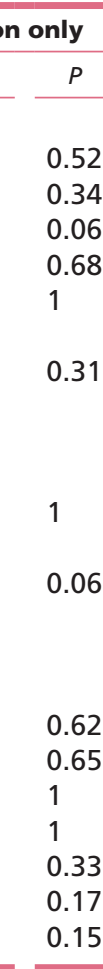

\begin{tabular}{|c|c|}
\hline \multicolumn{2}{|c|}{ RAS + TERT mutation } \\
\hline No. (percent) & $P$ \\
\hline 6 & \\
\hline $62(58-65)$ & 0.024 \\
\hline $4 / 6(66.7)$ & 0.044 \\
\hline $2 / 6(33.3)$ & 0.44 \\
\hline $4.4(2.4-6.2)$ & 0.22 \\
\hline 2/6 (33.3) & 0.28 \\
\hline $0 / 6(0)$ & $<0.001$ \\
\hline $1 / 6(16.7)$ & \\
\hline 1/6 (16.7) & \\
\hline $4 / 6(66.7)$ & \\
\hline $5 / 6(83.3)$ & 0.004 \\
\hline $1 / 6(16.7)$ & 0.09 \\
\hline $2 / 6(33.3)$ & \\
\hline $2 / 6(33.3)$ & \\
\hline $1 / 6(16.7)$ & \\
\hline $3 / 6(50)$ & 0.36 \\
\hline 2/6 (33.3) & 1 \\
\hline $2 / 2(100)$ & $<0.001$ \\
\hline $4 / 4(100)$ & $<0.001$ \\
\hline $6 / 6(100)$ & $<0.001$ \\
\hline $0 / 6(0)$ & 1 \\
\hline $25(23-40)$ & 0.87 \\
\hline
\end{tabular}

This table summarizes the analyses in 162 patients with PTC negative for BRAF V600E mutation. AJCC_M refers to distant metastasis status at the diagnosis of thyroid cancer; M1, presence of metastasis. The group 'Distant metastasis' below the group 'AJCC_M' in the table refers to any distant metastasis, including distant metastasis at the diagnosis and distant metastasis discovered during the follow-up time, i.e., metastatic recurrence. AJCC, American Joint Committee on Cancer; IQR, interquartile range. 
in fact, $R A S$ mutation was even inversely associated with lymph node metastasis $(P=0.043)$. In contrast, the coexistence of RAS and TERT promoter mutations was strongly associated with older patient age, male sex, late disease stages III and IV, distant metastasis and recurrence compared with the group negative for either mutation ( $P=0.024,0.044,0.004,<0.001$ and $<0.001$, respectively) (Table 3). Mortality could not be analyzed due to small number of deaths. In the analysis on CPTC, we similarly observed synergistic effects of the two mutations on poor clinicopathologic outcomes (Supplementary Table 7).

On the HR analysis for disease recurrence (Table 2), all 6 patients harboring both RAS and TERT promoter mutations had disease recurrence (100\%, 1173 recurrences per 1000 person-years; $95 \%$ CI, 526.99-2610.97) vs only 6/87 (6.9\%, 22.15 recurrences per 1000 person-years; 95\% CI, 9.94-49.25) in patients harboring neither mutation, corresponding to a HR of 39.04 (95\% CI, 10.56-144.3; $P<0.001)$. The HR remained significant at 106.76 (95\% CI, 15.30-744.49, $P<0.001)$ after adjustment for patient age and sex and at 89.88 (95\% CI, 8.89-909.14, $P<0.001)$ after additional adjustment for clinicopathologic risk factors and still significant at $138.0(95 \% \mathrm{CI}, 5.75-3313, P=0.002)$ after further additional adjustment for institution. Similar HR results for recurrence were obtained when only CPTC was analyzed (Supplementary Table 6).

We next performed Kaplan-Meier analyses of the impacts of RAS and TERT promoter mutations on patient survival and disease recurrence-free survival. With the limited number of deaths, no difference was seen in patient survival among the different genotypes (Fig. 2C). This was also the case when only CPTC was analyzed (Supplementary Fig. 2C). In contrast, although RAS or TERT promoter mutation, each alone, was associated with only a modest decline in recurrence-free survival curve, coexistence of the two mutations was robustly associated with a sharp decline in the recurrence-free survival curve (Fig. 2D). These results were similarly observed when only CPTC was analyzed (Supplementary Fig. 2D).

\section{Impacts of BRAF V600E/RAS or TERT promoter mutation alone or in coexistence on clinicopathologic outcomes of PTC in the TCGA data}

As $B R A F$ V600E and RAS mutations were mutually exclusive but were both associated with TERT promoter mutations in

Table 4 Impact of BRAF V600E/RAS or TERT promoter mutation or their coexistence on clinicopathologic outcomes of PTC.

\begin{tabular}{|c|c|c|c|c|c|c|c|}
\hline \multirow[b]{2}{*}{ Characteristic } & \multirow{2}{*}{$\begin{array}{l}\text { No mutation } \\
\text { No. (percent) }\end{array}$} & \multicolumn{2}{|c|}{ BRAFIRAS mutation only } & \multicolumn{2}{|c|}{ TERT mutation only } & \multicolumn{2}{|c|}{ BRAFIRAS + TERT mutation } \\
\hline & & No. (percent) & $P$ & No. (percent) & $P$ & No. (percent) & $P$ \\
\hline Sample size & 109 & 240 & & 5 & & 34 & \\
\hline Age at diagnosis, median (IQR) (years) & $46(33-59)$ & $45(34-54)$ & 0.34 & $49(37-68)$ & 0.51 & $66(58-72)$ & $<0.001$ \\
\hline Sex, male & $27 / 109(24.8)$ & $62 / 240(25.8)$ & 0.94 & $0 / 5(0)$ & 0.34 & $13 / 34(38.2)$ & 0.13 \\
\hline Multifocality & $53 / 103$ (51.5) & 107/237 (45.1) & 0.34 & $0 / 5(0)$ & 0.06 & 14/34 (41.2) & 0.33 \\
\hline Tumor size, median (IQR) (cm) & $2.9(1.9-4)$ & $2.4(1.5-3.5)$ & 0.09 & $4(3.0-5.0)$ & 0.68 & $3(2.4-4.6)$ & 0.12 \\
\hline Extrathyroidal invasion & $17 / 104(16.3)$ & $74 / 232(31.9)$ & 0.005 & $1 / 5(20)$ & 1 & $19 / 34(55.9)$ & $<0.001$ \\
\hline \multicolumn{8}{|l|}{ AJCC tumor stage } \\
\hline Stage I & $66 / 108$ (61.1) & $147 / 239$ (61.5) & 0.07 & $3 / 5(60)$ & 0.31 & $5 / 34(14.7)$ & $<0.001$ \\
\hline Stage II & $18 / 108$ (16.7) & 19/239 (7.9) & & $1 / 5(20)$ & & $3 / 34(8.8)$ & \\
\hline Stage III & 19/108 (17.6) & $53 / 239(22.2)$ & & $0 / 5(0)$ & & $11 / 34(32.3)$ & \\
\hline Stage IV & 5/108 (4.6) & $20 / 239(8.4)$ & & $1 / 5(20)$ & & $15 / 34(44.1)$ & \\
\hline Stage III + stage IV & 24/108 (22.2) & 73/239 (30.6) & 0.12 & $1 / 5(20)$ & 1 & 26/34 (76.4) & $<0.001$ \\
\hline \multicolumn{8}{|l|}{$\mathrm{AJCC}_{-} \mathrm{T}$} \\
\hline $\mathrm{T} 1^{-}$ & $31 / 107$ (29.0) & $78 / 240(32.5)$ & 0.08 & $1 / 5(20)$ & 0.06 & $5 / 34(14.7)$ & $<0.001$ \\
\hline $\mathrm{T} 2$ & 46/107 (43.0) & $74 / 240(30.8)$ & & $2 / 5(40)$ & & $8 / 34(23.5)$ & \\
\hline T3 & 30/107 (28.0) & $82 / 240(34.2)$ & & $1 / 5(20)$ & & $13 / 34(38.2)$ & \\
\hline $\mathrm{T} 4$ & $0 / 107(0)$ & $6 / 240(2.5)$ & & $1 / 5(20)$ & & $8 / 34(23.5)$ & \\
\hline $\mathrm{T} 3+\mathrm{T} 4$ & $30 / 107(28.0)$ & $88 / 240(36.7)$ & 0.14 & $2 / 5(40)$ & 0.62 & $21 / 34(61.7)$ & $<0.001$ \\
\hline AJCC_N, N1 & $38 / 92(41.3)$ & $107 / 218(49.1)$ & 0.26 & $3 / 5(60)$ & 0.65 & $19 / 33(57.6)$ & 0.21 \\
\hline AJCC_M, M1 & $0 / 54(0)$ & $3 / 146(2.1)$ & 0.56 & $0 / 4(0)$ & 1 & $4 / 21(19.0)$ & 0.005 \\
\hline Distant metastasis & $1 / 50(2)$ & $8 / 126(6.3)$ & 0.45 & $0 / 4(0)$ & 1 & $7 / 21(33.3)$ & $<0.001$ \\
\hline Tumor recurrence & $6 / 87(6.9)$ & 25/189 (13.2) & 0.15 & $1 / 5(20)$ & 0.33 & $13 / 25(52)$ & $<0.001$ \\
\hline Mortality rate & $3 / 108(2.8)$ & $5 / 232(2.2)$ & 0.71 & $1 / 5(20)$ & 0.17 & $5 / 34(14.7)$ & 0.019 \\
\hline Total follow-up, median (IQR) (month) & $30(17-46)$ & $35(19-60)$ & 0.18 & $13(6-28)$ & 0.15 & $42(17-52)$ & 0.23 \\
\hline
\end{tabular}

AJCC_M refers to distant metastasis status at the diagnosis of thyroid cancer; M1, presence of metastasis. The group 'Distant metastasis' below the group 'AJCC_M' in the table refers to any distant metastasis, including distant metastasis at the diagnosis and distant metastasis discovered during the follow-up time, i.e., metastatic recurrence.

AJCC, American Joint Committee on Cancer; IQR, interquartile range. 
PTC, we pooled the two mutations to collectively examine their relationship with TERT promoter mutations in affecting the clinicopathologic outcomes in the 388 PTC patients from the TCGA database. As shown in Table 4, in comparison with the group negative for any mutation, $B R A F \mathrm{~V} 600 \mathrm{E} / R A S$ mutation alone was only associated with extrathyroidal invasion $(P=0.005)$ and TERT promoter mutation alone was not significantly associated with any poor clinicopathologic characteristics. In contrast, the coexistence of BRAF V600E/RAS and TERT promoter mutation was strongly associated with older patient age, extrathyroidal invasion, advanced disease stages III/IV, high tumor stages T3 and T4, distant metastasis, disease recurrence $(P<0.001$ for all $)$ and patient mortality $(P=0.019)$. Similar results were obtained when only CPTC patients were analyzed (Supplementary Table 8).

We analyzed the HR of these genotypes for PTC recurrence (Table 2). Specifically, 13/25 patients harboring both BRAF V600E/RAS and TERT promoter mutations had disease recurrence $(52 \%, 184.2$ recurrences per 1000 personyears; 95\% CI, 106.96-317.28) vs only 6/87 (6.9\%, 22.15 recurrences per 1000 person-years; 95\% CI, 9.94-49.25) patients harboring no mutation, corresponding to a HR of 8.17 (95\% CI, 3.09-21.58; $P<0.001$ ). This HR remained significant at 12.44 (95\% CI, 3.33-46.56, $P<0.001)$ after adjustment for patient age and sex and still robustly significant at 13.16 (95\% CI, 2.39-72.42, $P=0.003$ ) after additional adjustment for classical clinicopathologic risk factors and at $14.71(95 \% \mathrm{CI}, 2.79-77.61, P=0.002)$ after further additional adjustment for institution. A similar robust HR of recurrence for coexisting BRAF V600E/RAS and TERT promoter mutations was observed when only CPTC was analyzed (Supplementary Table 6).

The HR for patient mortality was not significant for $B R A F$ V600E or TERT promoter mutation alone but was significant for the genetic duet of BRAF V600E and TERT promoter mutations $(P=0.038$; Supplementary Table 9$)$, consistent with a synergistic effect of the two mutations. Due to the low mortality of PTC and relatively small cohorts, HRs for mortality were not or only marginally significant for other genetic duet conditions. Similar results were obtained when only CPTC was analyzed (Supplementary Table 10). However, on Kaplan-Meier analyses, like the genetic duet of BRAF V600E and TERT promoter mutations (Fig. 2A and B), the genetic duet of $B R A F \mathrm{~V} 600 \mathrm{E} / R A S$ and TERT promoter mutations robustly affected the patient survival and disease recurrence-free survival curves. Specifically, as shown in Fig. 2E, although $B R A F$ V600E/RAS had no significant influence and TERT promoter mutation had limited cases, coexistence of BRAF V600E/RAS and TERT promoter mutations was significantly associated with a sharp decline in the patient survival curve. BRAF V600E/RAS or TERT promoter mutation alone had only a modest effect on disease recurrence-free survival, whereas coexistence of $B R A F$ $\mathrm{V} 600 \mathrm{E} / R A S$ and TERT promoter mutations was associated with a sharp decline in the disease-free survival curve (Fig. 2F). Similar results were obtained when only CPTC was analyzed (Supplementary Fig. 2E and F).

As for the genetic duet of BRAF V600E and TERT promoter mutations, a similar distribution pattern of the genetic duet of BRAF V600E/RAS and TERT promoter mutations was seen in the PTC subtypes, with a prevalence being $7 / 30$ (23.3\%), 23/270 (8.5\%) and 4/83 (4.8\%) in TCPTC, CPTC and FVPTC (Supplementary Table 5), respectively, corresponding to the aggressiveness order of TCPTC >CPTC $\gg$ FVPTC reported recently (Shi et al. 2016). These results were again consistent with an aggressive role of the genetic duet of BRAF V600E/RAS and TERT promoter mutations in PTC.

\section{Discussion}

Since the initial report of TERT promoter mutations in thyroid cancer three years ago (Liu et al. 2013), many studies have been devoted to investigating their role in the pathogenesis and clinicopathologic outcomes of thyroid cancer (Liu \& Xing 2016). An interesting aspect in this regard is the association of TERT promoter mutation with BRAF V600E, which, after its initial report (Liu et al. 2013), has been widely confirmed (Liu \& Xing 2016). We hypothesized that the coexisting event of these two major oncogenic mutations likely played a special role in thyroid cancer pathogenesis and conferred a subset of PTC unique clinicopathologic properties (Liu et al. 2013). Indeed, this was proven to be true in our subsequent studies, which demonstrated a robust synergistic role of coexisting BRAF V600E and TERT promoter mutations in the development of poor clinicopathologic outcomes of PTC, including sharply increased tumor recurrence and patient mortality (Xing et al. 2014a,b). As a result, we proposed that the genetic duet of BRAF V600E and TERT promoter mutations constitutes a unique genetic background that strongly promotes the aggressiveness of thyroid cancer and predicts the worst clinical outcomes of PTC, which was lauded by other investigators (Ngeow \& Eng 2014). These findings on the genetic duet of BRAF V600E and TERT promoter mutations were confirmed by other

Published by Bioscientifica Ltd. 
studies (Bullock et al. 2016, Jin et al. 2016, Kim et al. 2016, Song et al. 2016). Our recent study on an extended cohort of 1051 PTC patients again demonstrated a robust role of coexisting BRAF V600E and TERT promoter mutations in the aggressiveness of PTC and a strong prognostic value of this genetic duet for the mortality of PTC patients (Liu et al. 2016). Nevertheless, the potential role of RAS mutations, which are the second most common after BRAF V600E mutation in PTC, in this genetic interplay in affecting the aggressiveness of PTC has not been established.

Our recent meta-analysis on TERT promoter mutations in thyroid cancer demonstrated a significant association between RAS and TERT promoter mutations in FTC and poorly differentiated and anaplastic thyroid cancers (Liu \& Xing 2016). This was confirmed in two recent studies (Landa et al. 2016, Sohn et al. 2016). Marginal association of coexisting RAS and TERT promoter mutations with poor tumor behaviors was seen in limited FTC cohorts (Muzza et al. 2015, Sohn et al. 2016). A recent study reported an association between this genetic duet and poor clinical outcomes in a cohort of patients mixed with FTC and PTC, in which the genetic duet occurred mostly in the FTC patients (Song et al. 2016). Also, the potential influence of BRAF V600E was not dissected in this analysis. Thus, the specific role of coexisting RAS and TERT promoter mutations in the pathogenesis and clinical outcomes of PTC remains undefined. Importantly, because BRAF V600E and RAS mutations are mutually exclusive (Xing 2013) and the previous studies on the role of the genetic duet of BRAF V600E and TERT promoter mutations in PTC did not separate the potential influence of $R A S$ mutations, it is not clear whether the RAS mutation status could in fact affect the conclusions on the genetic duet of BRAF and TERT promoter mutations in the previous studies.

We performed the present study using the unique PTC cohort in the TCGA database to address the issues discussed previously. The TCGA thyroid cancer database provided an ideal cohort of PTC for the present study as it consisted of patients from a large number of medical institutions in the North America with comprehensive genetic, pathological and clinical information. The present study was therefore multicenter in nature. The first goal of this study was to confirm the previous findings in single-institution studies and validate the role of TERT promoter mutations and the genetic duet of $B R A F$ V600E and TERT promoter mutations in PTC. This was successfully achieved. Specifically, we demonstrated that BRAF V600E and TERT promoter mutations each alone had no or only a modest effect, but the genetic duet of the two had a robust effect on virtually all the poor clinicopathologic outcomes of PTC. Thus, the findings in previous studies on BRAF V600E and TERT promoter mutations were essentially all reproduced in this multicenter data analysis, confirming and validating the recent conclusions on the genetic duet of $B R A F \mathrm{~V} 600 \mathrm{E}$ and TERT promoter mutations in PTC. A similar pattern of the effects was seen for RAS and TERT promoter mutations. Specifically, RAS mutation alone had no effect, whereas the genetic duet of the two had a strong synergistic effect on the poor clinicopathologic outcomes of PTC. This was the case whether the whole PTC cohort or only CPTC was analyzed. This is the first demonstration of a robust synergistic role of coexisting RAS and TERT promoter mutations in the aggressiveness of PTC.

In addition to the multicenter nature, another unique strength that distinguishes the present study from previous studies is the separation of BRAF V600E and $R A S$ mutations from each other in the analysis of their synergistic effects with TERT promoter mutations. This provided definitive evidence supporting that coexistence of either BRAF V600E or RAS mutation with TERT promoter mutation is a robust genetic mechanism that drives the worst aggressiveness of PTC. The underlying molecular mechanism was proposed to involve the activation of the MAP kinase pathway, which promotes the TERT expression by upregulating ETS transcription factors acting at the mutation sites in the TERT promoter (Liu et al. 2013, Liu \& Xing 2016). This is consistent with the finding that coexisting BRAF V600E and TERT promoter mutations were associated with increased expression of TERT (Vinagre et al. 2013). It is not clear how the genetic duet occurs. As either BRAF/RAS mutation or $T E R T$ promoter mutation can occur individually and each alone has limited aggressive role, one possibility is that the occurrence of each of the two individual mutations is a random event independent of each other; but once the two individually random mutations happen to occur in the same thyroid cancer cell, the genetic duet confers the cell superior survival (and aggressiveness) ability and consequently, from an evolutionary perspective, such a cell can be preferentially selected naturally, resulting in the observed common concurrence of the two mutations in aggressive thyroid cancers.

A weakness of the present study is the relatively small cohort of 388 patients, which was smaller than many of the previous single-institution studies. Separation of $B R A F$ V600E from RAS mutations made some of the subgroup 
analyses even smaller. This may explain the marginal significance of multivariate analyses in some subgroups. This was particularly an issue when HRs for the relatively low mortality were analyzed. Nevertheless, this study was able to essentially reproduce all the previous findings on TERT promoter mutations and demonstrate a robust role of the genetic duet of TERT promoter and BRAF V600E or $R A S$ mutations in PTC, establishing a strong prognostic power for these genetic events in the aggressiveness of PTC. In fact, when the BRAF V600E and RAS mutation patients were pooled to analyze their synergistic effects with TERT promoter mutations, even more robust effects of the genetic duet of BRAF V600E/RAS mutations on poor clinicopathologic outcomes were observed (Table 4) and the effect on PTC recurrence remained significant even upon adjustment for all the conventional high-risk clinicopathologic factors (Table 2).

In summary, this study using the unique multicenter PTC cohort in the TCGA database confirmed the recent findings in single-institution studies on the role of $B R A F$ V600E and TERT promoter mutations in the aggressiveness of PTC. The role of the genetic duet of BRAF V600E and TERT promoter mutations was particularly firmly established by focused analyses on them with exclusion of RAS mutations. Importantly, this study for the first time established a similar role of the genetic duet of $R A S$ and $T E R T$ promoter mutations in PTC. The overall occurrence of coexisting BRAF V600E and TERT promoter mutations in a large series of PTC was 145/1892 (7.7\%) (Liu et al. 2016), and the overall coexisting BRAF V600E/RAS and TERT promoter mutations in the present study was $34 / 388(8.76 \%)$, which numerically correspond well to the conventionally known about $5-10 \%$ of PTC patients who inherently have a particularly aggressive disease course and are the source of virtually all PTC-related mortalities (Haugen et al. 2016). We recently proposed a four-genotype risk stratification system for PTC with a risk order of the genetic duet $>>>>B R A F$ V600E alone $=T E R T$ promoter mutation alone $>$ the wild type for both genes (Liu et al. 2016). Given the findings in the present study, this genetic prognostication system may now be modified into a six-genotype prognostic system by incorporating $R A S$ mutation also into it with a risk order of genetic duet of BRAF V600E/RAS mutation and TERT promoter mutations $>>>>B R A F \mathrm{~V} 600 \mathrm{E}=T E R T$ mutation alone $>R A S$ mutation alone=wild-type genes in papillary thyroid cancer. This simple but powerful genetic molecular prognostic system may help pinpoint the small subgroup of PTC patients with the highest aggressiveness risk for personalized precision treatment.

\section{Supplementary data}

This is linked to the online version of the paper at http://dx.doi.org/10.1530/ ERC-16-0402.

\section{Declaration of interest}

$M$ Xing receives royalties as co-holder of a licensed USA patent related to $B R A F$ mutation in thyroid cancer. Other authors declare no conflict of interest.

\section{Funding}

This study was supported by USA National Institutes of Health (NIH) grants R01CA113507 and R01CA189224 to M Xing.

\section{Author contribution statement}

$\mathrm{M} X$ conceived, designed and supervised the study. X S collected the data. $X S, R L$ and $M X$ performed the analysis and interpreted the results. $X S$ and $M X$ wrote the manuscript. $X S, R L$ and $M X$ revised and approved the manuscript.

\section{Acknowledgements}

The results published here are in part based upon data generated by the TCGA Research Network: http://cancergenome.nih.gov/.

\section{References}

Alzahrani AS, Alsaadi R, Murugan AK \& Sadiq BB 2016 TERT promoter mutations in thyroid cancer. Hormones and Cancer 7 165-177. (doi:10.1007/s12672-016-0256-3)

Bullock M, Ren Y, O’Neill C, Gill A, Aniss A, Sywak M, Sidhu S, Delbridge L, Learoyd D, de Vathaire F, et al. 2016 TERT promoter mutations are a major indicator of recurrence and death due to papillary thyroid carcinomas. Clinical Endocrinology 85 283-290. (doi:10.1111/cen.12999)

Cancer Genome Atlas Research Network 2014 Integrated genomic characterization of papillary thyroid carcinoma. Cell 159 676-690. (doi:10.1016/j.cell.2014.09.050)

Garcia-Rostan G, Zhao H, Camp RL, Pollan M, Herrero A, Pardo J, Wu R, Carcangiu ML, Costa J \& Tallini G 2003 ras mutations are associated with aggressive tumor phenotypes and poor prognosis in thyroid cancer. Journal of Clinical Oncology 21 3226-3235. (doi:10.1200/ JCO.2003.10.130)

Haugen BR, Alexander EK, Bible KC, Doherty GM, Mandel SJ, Nikiforov YE, Pacini F, Randolph GW, Sawka AM, Schlumberger M, et al. 20162015 American Thyroid Association Management Guidelines for adult patients with thyroid nodules and differentiated thyroid cancer: the American Thyroid Association Guidelines task force on thyroid nodules and differentiated thyroid cancer. Thyroid 26 1-133. (doi:10.1089/thy.2015.0020)

Horn S, Figl A, Rachakonda PS, Fischer C, Sucker A, Gast A, Kadel S, Moll I, Nagore E, Hemminki K, et al. 2013 TERT promoter mutations in familial and sporadic melanoma. Science 339 959-961. (doi:10.1126/science.1230062)

Howlader N, Noone A, Krapcho M, Miller D, Bishop K, Altekruse S, Kosary C, Yu M, Ruhl J, Tatalovich Z, et al. 2016 SEER Cancer Statistics Review, 1975-2013. Bethesda, MD, USA: National Cancer Institute. (available at: http://seer.cancer.gov/csr/1975_2013/). 
Based on November 2015 SEER data submission, posted to the SEER web site.

Huang FW, Hodis E, Xu MJ, Kryukov GV, Chin L \& Garraway LA 2013 Highly recurrent TERT promoter mutations in human melanoma. Science 339 957-959. (doi:10.1126/science.1229259)

Jin L, Chen E, Dong S, Cai Y, Zhang X, Zhou Y, Zeng R, Yang F, Pan C, Liu Y, et al. 2016 BRAF and TERT promoter mutations in the aggressiveness of papillary thyroid carcinoma: a study of 653 patients. Oncotarget 7 18346-18355. (doi:10.18632/oncotarget.7811)

Kim TH, Kim Y-E, Ahn S, Kim J-Y, Ki C-S, Oh YL, Kim K, Yun JW, Park W-Y, Choe J-H, et al. 2016 TERT promoter mutations and longterm survival in patients with thyroid cancer. Endocrine-Related Cancer 23 813-823. (doi:10.1530/ERC-16-0219)

Landa I, Ibrahimpasic T, Boucai L, Sinha R, Knauf JA, Shah RH, Dogan S, Ricarte-Filho JC, Krishnamoorthy GP, Xu B, et al. 2016 Genomic and transcriptomic hallmarks of poorly differentiated and anaplastic thyroid cancers. Journal of Clinical Investigation 126 1052-1066. (doi:10.1172/JCI85271)

Liu R \& Xing M 2016 TERT promoter mutations in thyroid cancer. Endocrine-Related Cancer 23 R143-R155. (doi:10.1530/ERC-15-0533)

Liu X, Bishop J, Shan Y, Pai S, Liu D, Murugan AK, Sun H, El-Naggar AK \& Xing M 2013 Highly prevalent TERT promoter mutations in aggressive thyroid cancers. Endocrine-Related Cancer 20 603-610. (doi:10.1530/erc-13-0210)

Liu X, Qu S, Liu R, Sheng C, Shi X, Zhu G, Murugan AK, Guan H, Yu H, Wang Y, et al. 2014 TERT promoter mutations and their association with BRAF V600E mutation and aggressive clinicopathological characteristics of thyroid cancer. Journal of Clinical Endocrinology and Metabolism 99 E1130-E1136. (doi:10.1210/jc.2013-4048)

Liu R, Bishop J, Zhu G, Zhang T, Ladenson PW \& Xing M 2016 Mortality risk stratification by combining BRAF V600E and TERT promoter mutations in papillary thyroid cancer: genetic duet of BRAF and TERT promoter mutations in thyroid cancer mortality. JAMA Oncology [in press]. (doi:10.1001/jamaoncol.2016.3288)

Muzza M, Colombo C, Rossi S, Tosi D, Cirello V, Perrino M, De Leo S, Magnani E, Pignatti E, Vigo B, et al. 2015 Telomerase in differentiated thyroid cancer: promoter mutations, expression and localization. Molecular and Cellular Endocrinology 399 288-295. (doi:10.1016/j.mce.2014.10.019)

Ngeow J \& Eng C 2014 TERT and BRAF in thyroid cancer: teaming up for trouble. Journal of Clinical Oncology 32 2683-2684. (doi:10.1200/ jco.2014.56.5614)

Shi X, Liu R, Qu S, Zhu G, Bishop J, Liu X, Sun H, Shan Z, Wang E, Luo Y, et al. 2015 Association of TERT promoter mutation 1,295,228 C $>$ T with BRAF V600E mutation, older patient age, and distant metastasis in anaplastic thyroid cancer. Journal of Clinical
Endocrinology and Metabolism 100 E632-E637. (doi:10.1210/jc.20143606)

Shi X, Liu R, Basolo F, Giannini R, Shen X, Teng D, Guan H, Shan Z, Teng W, Musholt TJ, et al. 2016 Differential clinicopathological risk and prognosis of major papillary thyroid cancer variants. Journal of Clinical Endocrinology and Metabolism 101 264-274. (doi:10.1210/ jc.2015-2917)

Siegel RL, Miller KD \& Jemal A 2015 Cancer statistics, 2015. CA: A Cancer Journal for Clinicians 65 5-29. (doi:10.3322/caac.21254)

Sohn SY, Park W-Y, Shin H-T, Bae JS, Ki C-S, Oh YL, Kim SW \& Chung JH 2016 Highly concordant key genetic alterations in primary tumors and matched distant metastases in differentiated thyroid cancer. Thyroid 26 672-682. (doi:10.1089/thy.2015.0527)

Song YS, Lim JA, Choi H, Won J-K, Moon JH, Cho SW, Lee KE, Park YJ, Yi KH, Park DJ, et al. 2016 Prognostic effects of TERT promoter mutations are enhanced by coexistence with BRAF or RAS mutations and strengthen the risk prediction by the ATA or TNM staging system in differentiated thyroid cancer patients. Cancer $\mathbf{1 2 2}$ 1370-1379. (doi:10.1002/cncr.29934)

Vinagre J, Almeida A, Pópulo H, Batista R, Lyra J, Pinto V, Coelho R, Celestino R, Prazeres H, Lima L, et al. 2013 Frequency of TERT promoter mutations in human cancers. Nature Communications 4 2185. (doi:10.1038/ncomms3185)

Xing M 2005 BRAF mutation in thyroid cancer. Endocrine-Related Cancer 12 245-262. (doi:10.1677/erc.1.0978)

Xing M 2007 BRAF mutation in papillary thyroid cancer: pathogenic role, molecular bases, and clinical implications. Endocrine Reviews 28 742-762. (doi:10.1210/er.2007-0007)

Xing M 2013 Molecular pathogenesis and mechanisms of thyroid cancer. Nature Reviews Cancer 13 184-199. (doi:10.1038/nrc3431)

Xing M, Alzahrani AS, Carson KA, Viola D, Elisei R, Bendlova B, Yip L, Mian C, Vianello F, Tuttle RM, et al. 2013 Association between BRAF V600E mutation and mortality in patients with papillary thyroid cancer. JAMA 309 1493-1501. (doi:10.1001/jama.2013.3190)

Xing M, Liu R, Liu X, Murugan AK, Zhu G, Zeiger MA, Pai S \& Bishop S 2014a BRAF V600E and TERT promoter mutations cooperatively identify the most aggressive papillary thyroid cancer with highest recurrence. Journal of Clinical Oncology 32 2718-2726. (doi:10.1200/ JCO.2014.55.5094)

Xing M, Liu R \& Bishop S 2014b TERT promoter and BRAF mutations cooperatively promote papillary thyroid cancer-related mortality. Thyroid 24 (Supplement 1) A-131-A-139. (doi:10.1089/thy.2014.2410. abstracts.sc)

Xing M, Alzahrani AS, Carson KA, Shong YK, Kim TY, Viola D, Elisei R, Bendlova B, Yip L, Mian C, et al. 2015 Association between BRAF V600E mutation and recurrence of papillary thyroid cancer. Journal of Clinical Oncology 33 42-50. (doi:10.1200/jco.2014.56.8253)

Received in final form 2 November 2016

Accepted 13 November 2016

Accepted Preprint published online 14 November 2016
๑ 2017 Society for Endocrinology Printed in Great Britain
Published by Bioscientifica Ltd 